\title{
« La musique... l'expérience même de l'appropriation impossible ». Quelques variations sur un thème de Jacques Derrida
}

\author{
Marie-Louise MALLET \\ escrituraeimagen@ffilos.ucm.es
}

\section{Resumen}

Desde sus comienzos, la relación de la filosofía con la música ha sido sin duda problemática. ¿Qué herencia nos ha dejado Jacques Derrida al respecto? Aunque no mantuvo ninguna tesis sobre la música «como tal», ha abierto un camino -otro camino- hacia la misma, hacia su escucha. Frente a la primacía óptico-háptica de la ontología y su logofonocentrismo, Derrida nos acerca, a través de la escucha, a la experiencia como apropiación imposible de la différance que encierra la variación modulada del material sonoro y, más allá de toda presencia, a la experiencia inaccesible de la luz de un duelo originario, donde, en la velocidad infinita de un presente sin pasado y siempre por venir, se entrecruzan la vida y la muerte, la memoria y la espera. La cadencia con la que termina el Concierto de Brandenburgo $n^{\circ} 5$ en Re Mayor de J. S. Bach y el finale de la Sonata en do menor de Schubert pueden ser dos bellos ejemplos para esa experiencia indecidible.

Palabras clave: música, fonocentrismo, différance, repetición, tonalidad, modulación, Bach, Schubert, Richter.

\begin{abstract}
From its beginnings, the relationship between philosophy and music has always been problematic. What is Derrida's legacy to us on this subject? Although he did not develop a thesis about music "as such", he opened up a path -another path- to music, and its listening. Against the optical-haptical primacy of ontology and its logo-phono-centrism, Derrida leads us, through this process of listening, to experi-
\end{abstract}


ence as impossible appropriation of the différance which entails the modulated variation of sonorous matter, and, beyond all presence, to the inaccessible experience of light of an original mourning which, at the infinite speed of a present with no past and yet to come, life and death, memory and wait, intertwine. The ending cadence of the first movement of J. S. Bach's Brandenburg Concerto No 5 in D major, and the Finale of Schubert's Sonata in $C$ minor are two fine examples of this undecidible experience.

Keywords: Music, phono-centrism, différance, repetition, tonality, modulation, Bach, Schubert, Richter.

En guise de prélude, quelques mots sur l'image qui fait l'affiche du colloque. Quand je l'ai découverte, j'ai pensé qu'elle était comme pré-accordée à mon propos. Ce n'est pas une photographie, mais une silhouette seulement, ce que Kierkegaard, dans Ou bien... Ou bien, nomme « tracés d'ombres ${ }^{1}$ », semblable à cette silhouette que dessine Dibutade, en suivant sur un voile (ou sur un mur) la trace de l'ombre portée de l'amant dont elle va être séparée. Vous vous souvenez de la place qu'occupe cette légende dans Mémoires d'aveugle et comment Jacques Derrida en fait l'emblème de sa pensée d'un aveuglement comme origine et condition insurmontable du dessin. Dibutade, en effet, ne voit pas son amant qu'elle dessine, elle ne voit que son ombre. Le dessin, à l'origine, est donc, dit Jacques Derrida, « une skiagraphia », une " écriture de l'ombre », un « tracé d'ombre », une représentation graphique liée à l'absence ou à l'invisibilité du modèle :

Comme si voir était interdit pour dessiner, comme si on ne dessinait qu'à la condition de ne pas voir, comme si le dessin était une déclaration d'amour destinée ou ordonnée à l'invisibilité de l'autre, à moins qu'elle ne naisse de voir l'autre soustrait au voir. [...] Elle écrit, donc elle aime déjà dans la nostalgie ${ }^{2}$.

Le dessin voudrait saisir l'insaisissable, garder une présence qui se dérobe, apaiser cette nostalgie, guérir cette douleur d'un impossible retour ou d'un retour douloureux de la perte. N'est-ce pas ce qui pousse Jacques Derrida lui-même, lui qui

\footnotetext{
1 «Tracés d'ombres » est le titre d'un chapitre de Enten-Eller, c'est-à-dire Ou bien...Ou bien, dans la traduction de F. et O. Prior et M.H. Guignot (Paris, Gallimard, 1949) ou L'Alternative, dans la traduction de P.-H. Tissot et E.-M. Jacquet-Tissot (Paris, Editions de l'Orante, 1970). "Tracés d'ombres » est la traduction exacte du danois Skyggerids, « contours ombreux ». Dans L'Alternative (et cette traduction du titre de l'ouvrage (Enten-Eller) en banalise le caractère insolite), la traduction par «Silhouettes» (renvoyant au nom d'Etienne de Silhouette), perd la richesse de sens du terme danois. 2 J. Derrida, Mémoires d'aveugle. L'autoportrait et autres ruines, Paris, Réunion des musées nationaux, 1990, p. 54.
} 
avoue avoir toujours été incapable de dessiner, à tenter l'impossible, dessiner le profil de sa mère mourante, et à garder ce qu'il nomme « l'archive de ce désastre ».

Or sa maladresse est un enseignement. Peut-être faut-il être, en effet, quelque peu maladroit pour apercevoir ce que l'adresse, au contraire, pourrait masquer. Ainsi, lorsqu'il se découvre « incapable de suivre de [sa] main la prescription d'un modèle », « comme si au moment de dessiner, je ne voyais plus la chose », dit-il, il découvre là une loi du dessin. Comment prétendre en effet regarder à la fois la chose, le modèle, et les traits que, de sa propre main, l'on trace en vue de capter la chose ? «Ne faut-il pas être aveugle à l'un ou à l'autre? Se contenter toujours de la mémoire de 1'autre 3 ?»

La « chose même » se dérobe toujours, « la perception appartient dès l'origine au souvenir $4_{\Downarrow}$. L'ombre, « détachée du présent de la perception », « tombée de la chose même qui se partage ainsi », l'ombre est « une mémoire simultanée » et la baguette avec laquelle Dibutade dessine est « un bâton d'aveugle », conclut-il 5 .

Revenons un instant à l'affiche. Skiagraphie, sans yeux donc. Il a ôté ses lunettes qu'il tient à la main. Peut-être a-t-il les yeux fermés. La pipe tenue dans sa main droite n'est pas, comme la cigarette, une invite à la conversation. Plutôt au silence, à la méditation. Peut-être est-il à l'écoute... Peut-être a-t-il été surpris et arrêté par un bruit, un son en train de s'évanouir et qu'il faut saisir aussitôt, car ce qui se donne à entendre se dérobe dans son surgissement même : ce que l'on n'a pas entendu ne reviendra plus, est perdu à jamais ; de ce qui semble revenir, nous ne pourrons jamais être sûrs que c'est le même, déjà venu. Les objets de l'œil sont, pour la plupart, stables, comparables dans la simultanéité de l'espace. Pour l'oreille, le temps ne suspends jamais son vol... il faut saisir au vol, «à la volée », ce qui se fait entendre. Seule la mémoire en garde la trace, comme une ombre qui, telle Eurydice, risque de disparaître dès lors que l'on tenterait de la fixer rétrospectivement.

Nous avons déjà commencé à parler de musique...

Le titre de ma communication cite, partiellement, et donc en en détournant quelque peu le sens, les derniers mots d'un entretien avec Elisabeth Weber, publié à la fin de Points de suspension :

La musique, s'il y en a, la musique, d'abord, je l'écoute. C'est l'expérience même de l'appropriation impossible. La plus joyeuse et la plus tragique6.

\footnotetext{
3 Ibid. p. 43. [Je souligne]

4 Ibid. p. 54. [Je souligne]

5 Ibid. p. 54.

6 Jacques Derrida, « Passages - du traumatisme à la promesse », dans Points de suspension. Entretiens, présentés par Élisabeth Weber, Paris, Galilée, 1992, p. 409.
} 
« La musique, s'il y en a... » La question d'Elisabeth Weber portait sur la pluralité des voix dans les textes de Jacques Derrida, qu'elle rapprochait, à juste titre, de la musique. Et elle lui demandait s'il n'avait jamais tenté d'écrire sur cette «multiplicité des voix en musique ». La réponse de Derrida concerne d'abord la musicalité des voix dans le texte. Ces voix, dit-il, il n'écrit pas « sur elles », il essaie de « les laisser parler ». Leur musique, «s'il y en a »- et, élégance ou modestie, il répète plusieurs fois ce «s'il y en a »-, « quand elle surgit et si elle surgit », elle lui arrive, dit-il : « à travers moi, sans moi, au-delà du contrôle que je pourrais avoir sur elles ». « La musique des voix, s'il y en a, dit-il encore, je ne la signe pas. » Et il conclut (ce sont les derniers mots de l'entretien et du livre) : « la musique, d'abord, je l'écoute. C'est 1'expérience même de l'appropriation impossible. La plus joyeuse et la plus tragique. ") Conclusion qui convient non seulement à cette polyphonie musicale des voix dans le texte, mais à la musique en général, si musique en général il y a.

Auparavant, au début de sa réponse, il avait dit :

Je me demande si la philosophie qui est aussi la naissance de la prose n'a pas signifié la répression de la musique ou du chant. La philosophie ne peut pas, en tant que telle, laisser le chant résonner en quelque sorte ${ }^{7}$

Comme un écho à cette exclamation de Nietzsche dans Le Gai savoir:

« De la cire dans les oreilles », c'était là, jadis, presque la condition préalable au fait de philosopher : un authentique philosophe n'avait plus d'oreille pour la vie, pour autant que la vie est musique, il niait la musique de la vie, - et c'est une très vieille superstition de philosophe que de tenir toute musique pour musique de sirènes ${ }^{8}$.

Dans les deux cas il est dit que la philosophie « en tant que telle » ne peut que se défier, réprimer, ou simplement ignorer, la musique.

Mais c'est la pensée de Jacques Derrida qui m'a aidée à m'approcher des raisons qui font qu'il ne peut qu'en être ainsi. Et puisque le motif du Colloque est l'héritage, les « héritages de Derrida », je voudrais tenter de dire, si peu et si mal que ce soit, quelque chose de ce legs, de ce que, s'agissant de musique, je dois à cette pensée.

Il a proclamé un jour, en 2002 : «Je n'ai à peu près jamais soufflé mot de la musique comme telle... ${ }^{9} »$ Cela est vrai de la musique « comme telle » - Et il sou-

\footnotetext{
7 Ibid., p. 408.

8 Nietzsche, Le Gai savoir, § 372, tr. Pierre Klossowski, dans Euvres philosophiques complètes V, textes établis par Giorgio Colli et Mazzino Montinari, Paris, Gallimard, 1982, p. 281.

9 Jacques Derrida, «Cette nuit dans la nuit de la nuit... », dans Rue Descartes, n ${ }^{\circ}$, novembre 2003. (Ce texte est la retranscription d'une communication faite au Collège International de Philosophie lors du « Samedi du livre » consacré à La Musique en respect (Paris, Galilée, 2002), le 1er février 2003).
} 
ligne d'italique ce « comme telle ». Outre qu'il se défie toujours du « comme tel» de l' " en tant que tel », il est vrai qu'il a moins parlé de musique que de littérature, d'architecture, de peinture, dessin ou même cinéma (mais a-t-il parlé de chacun de ces arts « en tant que tel », ou « comme tel »?...). Il n'y a pas de « thèse » de Jacques Derrida sur la musique, elle ne constitue pas une thématique de sa pensée : ni « thème » ni « thèse » la musique n'est pas, pour lui, un « ob-jet » de pensée, posé et immobilisé dans l'en face que requiert la théorie.

Mais s'il n'a pas proposé une nouvelle « philosophie » c'est-à-dire une « théorie », une « esthétique » de la musique, il a fait bien davantage et beaucoup mieux : ouvrir un chemin de pensée vers la musique, vers l'écoute de la musique, chemin que la philosophie, dans sa plus grande tradition, s'est barré, obstrué, en vertu d'une nécessité quasi structurelle.

Des quelques pas que j'ai pu faire sur ce chemin, grâce à lui, je ne dirai pas grand chose et, dans les limites de cette communication, je me bornerai à esquisser seulement quelques traits de ce qui, dans sa pensée, me semble ouvrir ce chemin.

« La philosophie en général [...] obéit au doigt et à l'œil... », lit-on dans Le toucher, Jean-Luc Nancy ${ }^{10}$ : par cet usage enjoué d'une expression triviale il condense de façon saisissante un long parcours de sa pensée. Tout d'abord la déconstruction du phono-logo-centrisme qui, depuis Platon au moins, et tout au long de son histoire, a structuré la démarche philosophique. Phonocentrisme qui, privilégiant la voix, la phoné, censée être présence pure, présence à soi du sujet dans sa parole, présence de la pensée à elle-même et enfin parole vivante adressée à l'autre dans le dialogue, relègue l'écriture au rang inférieur de technique auxiliaire, d'esclave susceptible de tromper, trahir, voire porter la mort à cette parole vive qu'elle est censée relayer et servir, en son absence. Derrida déconstruit cette illusion d'une présence immédiate du sens qui rend aveugle à cette archi-écriture qui, dans la parole même, avant toute écriture au sens banal du terme, inscrit la différance dans la présence, l'extériorité dans l'intériorité, la répétition dans l'immédiateté, la technique dans le vivant, la trace de l'autre dans le sujet parlant.

Ce phonocentrisme ne privilégie pas pour autant l'écoute, car il se double d'un logocentrisme. La voix, la phoné, n'intéresse la philosophie qu'en tant que voix qui parle, qui énonce une proposition, un sens intelligible : seul importe ce qu'elle dit, le « dit» et non le « dire », le « quoi » et non le « comment». Le philosophe est sourd à tout le reste. Le ton est sans importance pour lui (ou les changements de ton, ce «Wechsel der Töne » qui importe tellement à Jacques Derrida, sa «préoccupation principale... », dit-il dans La Carte postale11) . A fortiori, sont sans intérêt phi-

\footnotetext{
10 Jacques Derrida, Le toucher, Jean-Luc Nancy, Paris, Galilée, 2000, p. 139.

11 Jacques Derrida, "Envois », La Carte postale, de Socrate à Freud et au-delà. Paris, Aubier Flammarion, 1980, p. 217.
} 
losophique toutes les autres modalités de la voix, le cri, la voix de la plainte ou de l'exultation, la voix de la prière, le chant en ses infinies modulations, etc.... Pour la philosophie la phoné est avant tout logos : parole et raison, calcul, proportion... Et c'est ici que l'œil conquiert son privilège sur l'écoute. L'œil mais aussi la main. Certes, la parole ne se voit pas, ni ne se prend en main, mais toutes les catégories de la pensée philosophique transposent, dans le domaine de l'intelligibilité, les structures de la visibilité et de la préhension : qu'il s'agisse de l'ob-jet, de la perception de l'objet comme de ce qui est placé, jeté devant soi, donné à la vue, à la garde du regard, ou à portée de la main, Vorhanden ou Zuhanden, ou bien du concept (Begriff), il s'agit toujours de prendre (capere, percipere, concipere, prehendere, begreifen), de maîtriser dans une prise. De l'objet visible, du phénomène (phainesthai), c'est-à-dire ce qui brille et qui se donne à voir, à l'idée, l'eidos, il s'agit toujours de voir une forme: "l'eidos figure un contour de visibilité intelligible 12», écrit Jacques Derrida dans Mémoires d'aveugle, c'est « une visibilité qui a besoin de lumière 13 ». La forme définit, retient et enferme dans son contour ce qu'elle définit et à quoi elle donne prise. Qu'il s'agisse de l'intuition (de intueri, voir), de l'évidence (de videre, voir), de l'aletheia, la vérité comme " dévoilement », de la théorie ou contemplation (de theorein, regarder), en grec comme en latin, en français comme en allemand et dans toutes les langues de la tradition de la philosophie occidentale ce passage incessant du voir au prendre, de la garde du regard à la garde de la prise, assure le passage du sensible à l'intelligible, de la « présence » à la « re-présentation » et le « rassemblement» de tout le divers sous l'autorité du logos. Revenant, dans Politiques de l'amitié, sur ce logocentrisme qui l'occupe depuis ses premiers travaux, Derrida écrit :

$\mathrm{Au}$ fond, le logocentrisme, ce n'est peut-être pas tant le geste qui consiste à mettre le lógos au centre que l'interprétation du lógos comme Versammlung, c'est-à-dire le rassemblement qui précisément concentre ce qu'il configure ${ }^{14}$.

Enfin, dans Le toucher, Jean-Luc Nancy, revenant sur l'intuitionnisme philosophique, « constitutif de la philosophie même », sur le « privilège de la vue » qu'implique son nom même, il met alors l'accent sur le rôle du toucher dans cette configuration fondatrice de l'ontologie, la faisant apparaître comme non moins tributaire de configurations tactiles :

12 Jacques Derrida, Mémoires d'aveugle. L'autoportrait et autres ruines, op. cit., p. 22.

13 Jacques Derrida, «Penser à ne pas voir », dans Annali (Fondazione Europea del Disegno (Fondation Adami), Milan, Bruno Mondadori ed.), 2005/1 p. 65. [Ce texte semble la transcription d'une intervention orale prononcée vers 2000 environ].

14 Jacques Derrida, Politiques de l'amitié, Paris, Galilée, 1994, p. 378. 
Au moins depuis Platon, sans doute, et malgré son endettement auprès du regard, l'intuitionnisme est aussi une métaphysique et une tropique du toucher, une métaphysique comme hapto-tropique 15.

Ainsi, qu'il soit optique ou haptique (et en réalité il est toujours l'un et l'autre), l'intuitionnisme philosophique, c'est-à-dire la philosophie comme ontologie, vise toujours « un plein de présence immédiate ».

Or dans cette visée la musique ne peut trouver sa place. C'est pourquoi, de Platon à Hegel et au-delà, la musique a presque toujours été mal traitée (et maltraitée) par la philosophie.

Si l'on excepte les spéculations de type mathématique ou mathématico-cosmologique, auraient-elles, comme c'est le cas chez Leibniz, une incontestable portée métaphysique, il n'est pas exagéré de poser qu'entre philosophie et musique, tous comptes faits, il ne se sera pas passé grand-chose en plus de deux mille ans, et que l'histoire de leurs rapports est en définitive assez terne 16

écrit Philippe Lacoue-Labarthe, dans Musica ficta (et, s'agissant de musique, je saisis ici l'occasion de reconnaître ma dette envers lui, aussi). Même lorsqu'elle lui assigne une place élevée dans une hiérarchie des arts - et la philosophie, toujours, hiérarchise et toujours, depuis les pythagoriciens, en privilégiant, dans la musique, ce qui peut s'y réduire à des proportions mathématiques et donner prise à la théorie-, il vient toujours un moment où la philosophie manifeste à tout le moins un embarras, quand ce n'est pas un rejet violent ${ }^{17}$. Que faire en effet, que dire d'un art dont on ne sait jamais vraiment ce qu'il exprime ni même s'il exprime quelque chose, qui semble un jeu de formes, vides de sens mais qui, étrangement, ont des effets puissants et incontrôlables. Un art dont les œuvres ne se laissent pas rassembler dans la présence, dans l'immédiateté d'une présence pleine, mais qui peut cependant émouvoir comme aucun autre... Un art surtout qui semble échapper à la « représentation » qui, seule, donne prise à la réflexion philosophique. Ce désarroi ou ce rejet culminent, cela va de soi, lorsque la musique, cessant d'accompagner des paroles, devient musique « pure », musique purement vocale, sans parole ou sans parole intelligible ou, pis encore, musique purement instrumentale... Ainsi, pour Hegel, de même qu'il faut la statue du dieu dans le temple pour que l'architecture accède au sens, il faut le texte dans la musique pour que celle-ci ne se réduise pas à une architecture sonore, vide de sens et, qui plus est, une architecture sans consis-

\footnotetext{
15 Jacques Derrida, Le toucher, Jean-Luc Nancy, op. cit , p. 138

16 Philippe Lacoue-Labarthe, Musica ficta (figures de Wagner), Paris, Christian Bourgois, 1991, p. 165-166.

17 De La République et surtout des Lois de Platon, jusqu'à l'Esthétique de Hegel et au-delà, on pourrait voir se dérouler le même fil de cette longue histoire.
} 
tance, évanescente (une architecture toujours déjà en ruine), qui échappe à toute prise du concept. À l'écoute d'une musique privée du support d'un texte, des représentations qui donnent prise au concept, Hegel cherche vainement des « points d'appuis », ces Haltpunkte dont la quête quasi obsessionnelle émaille son parcours et qui lui font défaut désormais - le philosophe du savoir absolu, le maître du concept ne trouve alors plus rien à relever et donc plus rien à dire ${ }^{18}$.

Ainsi, imaginons Hegel à l'écoute de la musique que nous allons entendre.

C'est la cadence qui termine le premier mouvement du Concerto Brandebourgeois $N^{\circ}$, en ré majeur, BWV 1050, de Jean-Sébastien Bach, concerto pour trois instruments solistes : violon, flûte et clavier. Comme il fait à peu près pour tous les musiciens - Rossini excepté qui a droit à un traitement de faveur -, Hegel nomme Bach seulement en passant : " génie grandiose, authentiquement protestant », dit-il. Compositeur de musique religieuse donc, c'est-à-dire de la musique la plus liée à la « représentation », et la plus chargée de sens.

Purement instrumentale, pour clavier, la « cadence » que nous allons écouter est extraordinaire. D'abord par sa longueur : elle occupe à elle seule tout le dernier tiers du mouvement. Par son écriture, ensuite : vous entendrez le même motif répété, cité, re-cité, renversé, varié, non pas sur tous les tons, mais sur tous les degrés de la gamme, montant et descendant à l'intérieur d'une échelle étroite de hauteurs, à la fois même et autre à chaque tour : répétition et différence. Exemple de ce que Jacques Derrida nomme itérabilité : « le surgissement de l'autre (itara, en sanscrit) dans la réitération », car " une différence fait toujours dévier la répétition ». « L'inédit surgit, qu'on le veuille ou non, dans la multiplicité des répétitions 19. » Itération qui n'est donc pas contradictoire avec l'unicité de l'événement ${ }^{20}$.

Ce jeu itératif de la répétition et de la différence provoque une attente à l'issue longuement et intensément différée, « une accumulation d'énergie, de force émotive », « sans cause extérieure apparente », jusqu'au surgissement de l'événement : une véritable explosion de virtuosité folle, folle jusqu'au vertige... C'est ce que François Florand, cité par Pierre Boulez dans Relevés d'apprenti21, nomme « l'ivresse musicale » de Bach, dont cette cadence est loin d'être le seul exemple :

\footnotetext{
18 Dans La musique en respect, j'ai tenté une étude de cette sorte de panique qui s'empare de Hegel, à la fin de la partie de l'Esthétique qui traite de la musique, lorsqu'il s'agit de penser la « musique pure », purement instrumentale, «die selbständige Musik».

19 Jacques Derrida, «Autrui est secret parce qu'il est autre » - Propos recueillis par Antoine Spire, Le Monde de l'éducation, $\mathrm{N}^{\circ} 284$, Septembre 2000, p. 14.

${ }^{20}$ Cf. « Signature événement contexte » dans Marges - de la philosophie, Paris, Minuit, 1972, p. 389.

21 Pierre Boulez, « Moment de Jean-Sébastien Bach » dans Relevés d'apprenti, (Textes réunis et présentés par Paule Thévenin), Paris, Le Seuil, 1966, p. 23-24.
} 
[...] il vient un moment où à force de tourner et retourner son motif, la tête semble tourner à l'auteur lui même. «Es schwindelt... ». Et c'est cela le sommet de l'œuvre. [...] En cela, si l'on veut, Bach est un possédé 22 .

Bach dionysiaque, Bach bacchique, en quelque sorte ! Aussi loin que possible de toute re-présentation, cette musique peut être entendue comme une assomption glorieuse de la différance...

Nous allons écouter cette cadence, jouée par Sviatoslav Richter. Un autre possédé !

À un ami qui lui signalait un article sur lui ayant pour titre : " Son Dieu, c'est Schubert ! », Richter répliqua aussitôt : « Mon Dieu, c'est la DIFFÉRENCE ! ${ }^{23}$ 》« différence » écrit en lettres capitales, sans doute pour traduire la force avec laquelle le mot fut prononcé, souligné de la voix. Qu'a-t-il voulu dire ? Je vous laisse rêver... Les propos de Richter sont souvent fulgurants ! Et, ici, non sans affinité, peut-être, avec cet autre propos, fulgurant aussi, de Jacques Derrida, dans La Carte postale : «Ton [cet autre nom de la différence, souligné ici en italique], c'est pour moi le nom de Dieu, mon Dieu, celui que je ne trouve pas... ${ }^{24} »$.

Écoute ... 25 [c'est un enregistrement de concert, comme presque toujours avec Richter qui, à la différence de Glenn Gould, n'aimait pas les studios]

Je voudrais maintenant tenter de mettre en rapport deux « textes », plus hétérogènes encore l'un à l'autre que ne le sont Robinson Crusoé de De Foe et le Séminaire de Heidegger, Les Concepts fondamentaux de la métaphysique. Monde, Finitude, Solitude 26 que Jacques Derrida fait se lire l'un l'autre tout au long de son dernier séminaire : il s'agit de quelques pages du deuxième volume du Séminaire La bête et le souverain 27 (p. 86 à 91), donc, et du dernier mouvement d'une sonate

\footnotetext{
22 François Florand, Jean-Sébastien Bach - L'œuvre d'orgue, Paris, Editions du Cerf, 1947, p. 125126

23 «Le bloc-notes de Richter (suite et fin) », dans Le Monde de la Musique, № 82, octobre 1985. Cet entretien avec Éric Anther est la suite de "Richter en quelques notes ", paru dans le N ${ }^{\circ}$ 80/81, août 1985.

24 Jacques Derrida, «Envois », dans La Carte postale, op. cit., p. 125.

25 Jean-Sébastien Bach, Concerto Brandebourgeois $N^{\circ} 5$ en ré majeur, BWV 1050, Sviatoslav Richter, piano, Oleg Kagan, violon, Marina Vorozhtsova, flûte, Orchestre de chambre du Conservatoire de Moscou, dir. Yuri Nikolaevsky, Live Moscou, 7/12/1978 (Oleg Kagan Edition, Vol. XXIV, Live Classics 2000).

26 Martin Heidegger, Les Concepts fondamentaux de la métaphysique. Monde, Finitude, Solitude, texte établi par Friedrich-Wilhem von Herrmann, tr. fr. Daniel Panis, Paris, Gallimard, 1992.

27 J. Derrida, Séminaire. La bête et le souverain. Volume II (2002-2003), Michel Lisse, Marie-Louise Mallet et Ginette Michaud (éds), Paris, Galilée, 2010, « Deuxième séance. Le 18 décembre 2002 », p. 86 à 91.
} 
de Schubert, la Sonate en ut mineur, D. 958. Tentative probablement vouée à l'échec, mais je tente cet impossible.

Je précise tout de suite cependant qu'il ne s'agit pas, pour moi, de suggérer un rapport de traduction de ces deux textes entre eux. D'autre part, pour ce que je voudrais tenter de dire, il faudrait pouvoir parler de façon polyphonique, et surtout pouvoir lire le texte en même temps qu'écouter la musique... toutes choses impossibles, surtout dans le cadre étroit du temps imparti. Enfin, je ne tenterai pas un véritable commentaire du texte de Jacques Derrida - Ginette Michaud en a publié un, attentif, minutieux et inspiré, auquel je vous renvoie 28 .

De ce texte bouleversant, je me limiterai seulement à faire entendre quelques uns de ses motifs majeurs, ceux qui me semblent le plus en résonance avec la musique, bouleversante elle aussi, à laquelle il s'est, pour moi, tellement lié que lisant le texte, j'entends la musique, écoutant la musique, j'entends le texte. Si ce n'est là qu'une alliance subjective, je vous demanderai de me le pardonner.

Écoutons donc d'abord le texte : il vient d'évoquer ce moment où Robinson, découvrant sur son île l'empreinte d'un pas, est envahi par une véritable terreur, incapable qu'il est de savoir si c'est la trace du pas d'un inconnu, d'un possible ennemi, ou la trace de son propre pas, la trace d'un passage passé oublié. « Il croit qu'il va bientôt mourir, qu'il court après sa mort ou que la mort lui court après ", écrit Derrida et c'est alors qu'il cite deux vers de John Donne29 :

Vous connaissez ce vers sublime et infini de John Donne, qui revient de je ne sais plus où à ma mémoire :

\section{I run to Death and Death meets me as fast \\ And all my Pleasures are like Yesterday}

Je cours vers la mort, je me précipite vers la mort et la mort vient à ma rencontre tout aussi vite. (Je cours sus à la mort, je cours à mort (I run to Death) et mort me vient dessus, mort de rencontre me saisit, m'attrape ou me rattrape aussi vite, me rattrape à la même vitesse, aussi tôt ${ }^{30}$.)

Une remarque : ce texte de Jacques Derrida est un texte posthume, non seulement au sens où, comme il l'écrit, « tous les écrits sont posthumes [...] même ceux qui sont connus et publiés du vivant de l'auteur 31 », mais au sens où, écrit à l'approche de sa mort, il n'est publié qu'après celle-ci. La sonate de Schubert est, elle

28 Ginette Michaud, «Courir à toute vietesse. Note télégraphique sur un poème de pensée de J. D. », Mosaic, «2. A Proceedings Issue. Following Derrida: Legacies », vol. 40, no 2, juin 2007, p. 56-62. 29 Il s'agit du premier des Holy Sonnets de John Donne (1572-1631). Jacques Derrida cite l'édition des Poèmes choisis (tr. fr., introd. et notes par Pierre Legouis, Paris, Aubier-Montaigne, 1955), mais il retraduit lui-même ces vers.

30 J. Derrida, Séminaire. La bête et le souverain. Volume II, op. cit. p. 86.

31 Ibid., p. 294. 
aussi, une des trois dernières sonates, dites «posthumes », composée à peine trois mois avant sa mort. Cependant, pas plus que Derrida, Schubert ne pensait que la mort était si proche et l'un comme l'autre faisaient alors des projets d'avenir. Enfin, comme Derrida aussi, Schubert aurait pu dire : « je posthume comme je respire 32 » : l'imminence de la mort a toujours été présente dans sa musique, il en « hume» depuis toujours l'approche, « anticipant sans anticiper, sentant au sens où l'on dit que les bêtes sentent [...] quand les bêtes sentent venir les catastrophes que nous ne voyons pas venir ${ }^{33}$ ». Peut-être, cependant, dans ces deux textes ultimes, le pressentiment est-il plus pressant.

Mais revenons au texte de Jacques Derrida. Ces deux vers de John Donne sont, comme en musique, les deux motifs sur lesquels s'élabore tout le cours de l'écriture, avec variations, retours, réexposition, développements, entrelacements réciproques.

Je relis. Énonciation du premier motif, donné par le premier vers qu'il traduit, non sans déjà le moduler quelque peu :

Je cours vers la mort, je me précipite vers la mort et la mort vient à ma rencontre tout aussi vite.

\section{Motif immédiatement repris :}

(Je cours sus à la mort, je cours à mort (I run to Death) et mort me vient dessus, mort de rencontre me saisit, m'attrape ou me rattrape aussi vite, me rattrape à la même vitesse, aussi tôt.)

Il ne s'agit pas d'une simple répétition cependant, mais d'une véritable modulation au sens musical, d'une variation modulée, dans un autre ton (ce "Wechsel der Töne », qualifié de « préoccupation principale... » dans La Carte postale ${ }^{34}$ ), autre ton qui colore autrement la première énonciation du motif, si bien que disant apparemment le même, cette modulation dit autre chose car elle dit autrement (vous entendrez nombre de ces sortes de modulations dans la sonate de Schubert).

Puis, aussitôt, le deuxième motif, la traduction du deuxième vers, motif qui, ainsi que le premier, joue des deux langues :

Et tous mes plaisirs sont comme hier, like Yesterday,

\footnotetext{
32 J. Derrida, « Circonfession », dans Jacques Derrida, avec G. Bennington, Paris, Le Seuil, 1991, §5, p. 28.

33 J. Derrida, Séminaire. La bête et le souverain. Volume II, op. cit. p. 249.

34 Jacques Derrida, « Envois », La Carte postale... op. cit ., p. 217.
} 
Motif énoncé et sitôt déjà repris, modulé, développé :

comme l'hier, comme venus d'hier, mes plaisirs sont déjà d'hier, mes plaisirs sont l'hier même, d'avance ils sont datés - et d'hier.

Motif varié et modulé : l'introduction de «d'avance » (que je souligne) va appeler tout un développement, où, avec la même liberté que celle d'un musicien composant sur le motif venu d'un autre une tout autre musique, Jacques Derrida va composer, lui aussi, une musique assez différente de celle de John Donne, me semble-t-il :

D'avance ils ont passé, ils sont passés, déjà passés dépassés, déjà des mémoires de jouissance révolue ou des revenances de plaisir. Mes plaisirs présents sont au présent (are) des présents d'hier, ils sont hier. Non pas : ils ont été ou ils furent hier, mais ils sont présentement hier. Leur être présent est hier, l'hier 35.

Dès lors les deux motifs vont se développer l'un par l'autre. C'est ce présent du plaisir comme hier, la vertigineuse simultanéité du présent et d'hier, simultanéité qui implique une vitesse infinie, qui relance le motif de la course et de la course à mort, faisant se chevaucher futur et futur antérieur :

C'est comme si présentement j'étais déjà mort, la mort venant si vite à ma rencontre et moi à la sienne, et on ne sait pas si je vais plus vite qu'elle vers elle ou elle plus vite que moi vers moi, nous courons l'un sur l'autre, comme pour savoir qui arrivera le premier, et au moment d'une rencontre qui ne se fait jamais attendre, on ne sait pas qui sera, qui aura été, hier, arrivé le premier ou la première, plus vite en tout cas que la vie, une vie que ce double mouvement accéléré gagne de vitesse, gagnant ainsi le temps de vitesse, gagnant même la vitesse de vitesse, vitesse au-delà de la vitesse, vitesse emportant la vitesse, allant plus vite que le temps et que la vitesse même, gagnant le temps de vitesse, si vite que ce que je vis au présent, voire ce que j'attends de l'avenir, est déjà passé, déjà mémoire et mélancolie, ou nostalgie (Heimweh). Voilà ce que voudrait dire, chaque fois que je le dis : la vie aura été si courte. Précipitation ou accélération incalculable, incommensurable, en avance sur elle-même - et gagnant le temps de vitesse.

Alors, évidemment, I run to Death peut vouloir dire à la fois « je cours vers la mort, je me presse vers la mort, je me précipite en direction de la mort qui vient à moi aussi vite ", mais aussi « je cours à mort », je cours comme un fou, à perte de souffle, pour fuir la mort, je cours à mort pour éviter la mort, je cours au-devant de la mort pour qu'elle ne me surprenne pas, pour prendre les devants, or elle me rattrape aussitôt ; mais « rattraper » ne va pas ici pour " meet », il faut donc supposer que même si je cours à mort, au-devant de la mort, devant la mort pour la fuir, la mort est là devant moi, elle m'attend déjà, à Samarkand, à la fois parce qu'elle va plus vite et parce $<$ que, $>$ venant au-devant de moi, prenant les devants, elle me devance, elle m'attend et vient à ma ren-

35 J. Derrida, Séminaire. La bête et le souverain. Volume II, op. cit. p. 87. [Je souligne] 
contre à l'instant même où je cours à mort - à la fois pour la fuir et pour la rejoindre. $A$ la fois pour la fuir et pour la rejoindre [...] Toujours antérieure, dans sa futurité même, comme ce qui reste à venir $[\ldots]^{36}$.

Non sans quelque possible analogie avec le développement qui au centre du mouvement de Schubert suspend un temps le tempo de la course, suit, dans le texte, un long développement, au tempo plus réflexif, sur l'hier, sur l'hiereté de l'hier que, faute de temps je ne citerai que partiellement. Cette course à mort et à la mort et sa « vitesse absolue », « sans différentiel de vitesse », « vitesse infinie » :

[...] ne fait pas seulement que mes plaisirs présents me sont présentement et d'avance dérobés, passés, déjà révolus dans leur présent même, déjà datés d'hier dans leur présent et leur ici-maintenant. Non, il faudrait dire et penser au contraire, $[\ldots]$ en prenant en compte la grammaire d'une proposition qui ose faire de hier un nom, l'hier, et non un adverbe [...] il faudrait accentuer et faire trembler le sens du comme (like), en commençant à entendre non pas seulement que mes plaisirs sont toujours affectés de péremption et d'avance datés, datés d'hier, passés et empreints de nostalgie, comme si on pensait d'abord l'essence du plaisir présent et qu'on s'apercevait ensuite, dans un second temps, le temps prédicatif, que ce que nous connaissons et éprouvons sous ce nom de plaisir ou de jouissance et comme plaisir présent se trouve ensuite, par-dessus le marché et sitôt que ce soit, déterminé comme passé, affecté de passé, et d'hiereté. Non, c'est le contraire, the other way round, si je puis dire, c'est hier qui donne le plaisir, le plaisir est hier, comme hier, il commence maintenant par être hier, non seulement à la manière d'hier mais en tant qu'hier.

\section{Et il va tourner et retourner ce motif dans lequel je dois couper ... :}

Je n'ai de plaisir $[\ldots]$ que parce que le plaisir est originairement hier, $[\ldots]$ il est dans son essence (Wesen), une Gewesenheit, un être-ayant-été, et c'est la nostalgie d'hier, d'une mort déjà arrivée, d'un deuil originaire, c'est cette nostalgie qui ne vient pas après le plaisir, mais qui, seule, me donne le plaisir et me le donne comme hier. Je ne jouis pas d'un plaisir d'abord présent qui se trouve aussitôt passé, nostalgique, endeuillé, non, le plaisir ne naît que du deuil, de la jouissance comme deuil. [...] du deuil de moi-même. Je suis depuis hier, [...] seul hier m'aura donné, seule ma mort ou le sentiment de ma mort, d'une mort qui m'aura gagné de vitesse, seule ma mort me laisse jouir et prendre du plaisir - en ce moment même. Seul l'hier absolu [...] fait présent au présent de la jouissance comme jouissance hiernale, si je puis dire. Sans le deuil, et le deuil de moi-même, le deuil de mon « je suis présent », il n'y aurait pas de plaisir. Il n'y aurait même pas de « je suis », de conscience, de cogito, de je pense ou de jouissance présente [...] mes plaisirs sont hier, ils sont l'hier, [...] je n'en jouis que comme mémoire ; et encore : mémoire et passé sont des concepts trop larges et vagues. L'hier, ce n'est pas seulement le passé dont

36 Ibid., p. 87-88 [Je souligne. J.D. souligne seulement « $\grave{A}$ la fois »]. 
je garde ou perds la mémoire, hier c'est le jour d'avance, le jour qui vient juste de passer, dont la lumière phénoménale vient de sombrer. Hier, c'est l'imminence passée d'aujourd'hui même, l'imminence du point du jour, le point du jour qui donne le jour au jour. [...] Ce qui naît naît comme mort-né. Mes plaisirs sont mort-nés ${ }^{37}$.

J'arrête ici la lecture. Pressée par le temps j'ai dû faire aussi quelques coupures dans le texte lu, coupures que je regrette car ici, comme en musique, la temporalité du texte, ses retours, ses reprises, ses progressives modulations sont inséparables du sens.

Il s'agit donc, dans ce texte, de ce que Jacques Derrida nomme survie, de cette « terminable survie depuis laquelle "je me vois vivre" traduit "je me vois mourir"... 38 », selon les mots de « Circonfession ». De cette survie dont il dit aussi, dans Apprendre à vivre enfin, que son « sens ne s'ajoute pas au vivre et au mourir », mais qu' « elle est originaire : la vie est survie 39 ». Il ne faut donc pas l'entendre seulement au sens courant, c'est-à-dire au sens de « continuer à vivre » (comme on peut survivre, par exemple, à une épreuve, un accident, une maladie, voire un massacre collectif, qui auraient pu être mortels), ou encore « vivre après la mort », (comme un livre, une œuvre, un enfant peuvent survivre à leur auteur). Non, « nous sommes structurellement des survivants », dit-il encore, en dernière page du texte.

Enfin et surtout, insiste-t-il, il ne faut pas penser « la survivance » comme étant « plutôt du côté de la mort 40 »:

Tout ce que je dis - depuis Pas, au moins, dans Passages - de la survie comme complication de l'opposition vie/mort; procède chez moi d'une affirmation inconditionnelle de la vie. La survivance c'est la vie au-delà de la vie, la vie plus que la vie [...] c'est la vie la plus intense possible 41 .

" doubler chaque seconde, comme une voiture l'autre, [...] la dédoubler plutôt $[\ldots]^{42}$ » écrivait-il dans le même passage de «Circonfession ».

Mais ne pourrait-on pas dire de même que la musique est structurellement survie ? « La matière sonore [...] confère un certain caractère tragique à toute musique», dit Daniel Barenboim, dans un entretien avec Edward Said43, car « le son [...] tend vers le silence». La musique est hantée par la perte : elle est sans cesse en

37 Ibid., p. 88-90. [Je souligne]

38 J. Derrida, « Circonfession », op. cit., §7, p.41

39 Jacques Derrida, Apprendre à vivre enfin, Paris, Galilée, 2005, p. 26.

40 Ibid., p. 54

41 Ibid., p. 54-55.

42 J. Derrida, « Circonfession », op. cit., §7, p.41

43 Edward W. Said et Daniel Barenboim, Parallèles et Paradoxes - Explorations musicales et politiques, tr. Ph. Babo, Paris, Le serpent à plumes, 2003, p. 157 et 198. 
train de tenter de maintenir en vie quelque chose qui menace de mourir à chaque instant.

Pour finir, donc, je vous propose d'écouter cet effort désespéré de survie qu'est le finale de la Sonate en ut mineur de Schubert.

C'est un Allegro (allegro désignant ici son tempo, extrêmement rapide, non sa Stimmung qui n'a rien d'allègre) : « cavalcade effrénée [...] effrayante par sa dimension et son déchaînement ininterrompu ", écrit Brigitte Massin dans son grand livre sur Schubert 44 . Cheval ou cavalier, celui qui court ainsi a la mort aux trousses et la mort en face, cette mort qui l'attend « à Samarkand ». Course à mort sur un rythme syncopé traversant, comme éperdu, toute une gamme de tonalités, avec des alternances de mineur et de majeur, changements de tonalités et de modes qui font qu'à chaque tour, l'un semblant refléter l'autre, la course semble se souvenir d'elle-même et que l'aujourd'hui semble se teinter de hiereté.

Remarquable par exemple, au début, le passage du motif de l'ut mineur à l'ut majeur. Comme souvent chez Schubert, le passage du mineur au majeur, s'il produit un effet d'éclaircissement, s'accompagne aussi, étrangement, d'un effet de lointain, comme si, du présent, on passait au souvenir et, plus encore, au souvenir comme « mémoire d'un passé qui n'a pas été présent 45 », selon le mot de Jacques Derrida - lumière d'un deuil originaire. Lointain lumineux inaccessible car toujours déjà perdu. Lumière endeuillée particulièrement sensible encore dans le long développement central qui, débutant lui aussi en majeur (si majeur) et entrelaçant au motif initial de la course un autre motif qui en suspend quelque peu le rythme syncopé, pourrait correspondre, répondre plutôt - je l'ai indiqué tout à l'heure -, à ce motif de « l'hier », de l' « hiereté » de l'aujourd'hui, de « l'imminence passée d'aujourd'hui même ».

La mort aux trousses, la mort en face, la course tente éperdument de lui échapper mais, "toujours antérieure, dans sa futurité même », la mort ne manque pas de rappeler qu'elle attend déjà, à Samarkand, par quelques coups d'accord de ré bémol (accords à vide, le plus souvent, accords d'octave), assénés avec violence (indiqués fortissimo), comme des verdicts implacables, jusqu'à « l'arrêt de mort » final qui exécute la sentence et met fin à la course.

Mais j'arrête là : je n'ai ni le temps, ni surtout la compétence pour pousser plus loin l'analyse. Surtout, je n'entends pas faire de cette musique un commentaire ou une traduction du texte de Jacques Derrida : ce serait absurde. Simplement la faire entendre comme une possible compagne. La faire écouter avec une oreille ouverte par le texte.

\footnotetext{
44 Brigitte Massin, Franz Schubert, Paris, Fayard, 1993 nouvelle édition, p. 1280.

45 « Passages - du traumatisme à la promesse », dans Points de suspension, op. cit., p. 396.
} 
Nous l'écouterons, si vous le voulez bien, interprétée, à nouveau, par Sviatoslav Richter. Toujours en concert. Du concert, « vie plus que la vie » pour lui, traversée périlleuse qui suppose que, tel Boutès ${ }^{46}$, on « se jette à corps perdu dans la mer 47 », expérience la plus intense de l'extrême urgence, il disait : « Il faut croire, mieux que saint Pierre, que l'on va marcher sur les eaux. Si l'on ne croit pas, on coule, et tout de suite ${ }^{48}$. » Une course à mort aussi, en quelque sorte.

Mais avant de l'écouter, je laisserai le dernier mot à Jacques Derrida qui, au début de la Sixième séance du Séminaire, parle du « courage de la pensée ». Du courage de la pensée comme « courage de la peur » : «pas d'absolu du courage, et du cœur, sans la panique absolue $49 »$, dit-il.

Écoute... 50

46 Cf. Pascal Quignard, Boutès, Paris, Galilée, 2008.

47 Bruno Monsaingeon, Richter. Écrits, conversations, Van de Velde-arte-Actes Sud, 1998, p. 373.

48 Ibid., p. 141.

49 J. Derrida, Séminaire. La bête et le souverain. Volume II, op. cit., « Sixième séance. Le 12 février 2003 », p. 215-216.

50Schubert, Sonate $N^{\circ} 19$ en ut mineur, Op. posth. (D. 958), "Allegro », par Sviatoslav Richter, Munich 1973, CD Olympia . 